\title{
Effects of an energy-restricted diet rich in plant-derived $\alpha$-linolenic acid on systemic inflammation and endothelial function in overweight-to-obese patients with metabolic syndrome traits
}

\author{
Sarah Egert ${ }^{1 *}$, Andrea Baxheinrich ${ }^{2}$, Young Hee Lee-Barkey ${ }^{3}$, Diethelm Tschoepe $^{3}$, \\ Ursel Wahrburg ${ }^{2} \dagger$ and Bernd Stratmann ${ }^{3} \dagger$ \\ ${ }^{1}$ Department of Nutrition and Food Science, Nutritional Physiology, University of Bonn, Endenicher Allee 11-13, \\ 53115 Bonn, Germany \\ ${ }^{2}$ Department of Human Nutrition, University of Applied Sciences, 48149 Muenster, Germany \\ ${ }^{3}$ Heart and Diabetes Center NRW, Rubr-University Bochum, 32545 Bad Oeynhausen, Germany
}

(Submitted 10 May 2014 - Final revision received 11 June 2014 - Accepted 13 June 2014 - First published online 2 September 2014)

\section{Abstract}

Plant-derived $\alpha$-linolenic acid (ALA) may reduce the risk of CVD, possibly by decreasing systemic inflammation and improving endothelial function. In the present study, the effects of a hypoenergetic diet rich in ALA $(3.4 \mathrm{~g} / \mathrm{d})$ on the biomarkers of systemic inflammation and vascular function were investigated in eighty-one overweight-to-obese patients with metabolic syndrome traits in comparison with a hypoenergetic diet low in ALA $(0.9 \mathrm{~g} / \mathrm{d}$, control). After a 6-month dietary intervention, there were significant decreases in the serum concentrations of C-reactive protein (CRP), TNF- $\alpha$, IL- 6 , soluble intercellular adhesion molecule-1 (sICAM-1), soluble endothelial selectin (sE-selectin) and asymmetric dimethylarginine in both dietary groups. However, no inter-group differences were observed for all these changes. The serum concentration of YKL-40 (human cartilage glycoprotein 39 or chitinase-3-like protein 1) decreased after the ALA diet when compared with the control diet $(P<0.05$ for time $\times$ treatment interaction). Plasma concentrations of fibrinogen did not significantly change in the two dietary groups. The decreases in the serum concentrations of sICAM- 1, sE-selectin, CRP and YKL- 40 were significantly correlated with the decreases in body fat mass. In conclusion, the present study indicates that in overweight-to-obese patients with metabolic syndrome traits, both vascular function and inflammation are improved during body-weight loss. The high ALA intake led to a more pronounced reduction in the serum concentration of YKL- 40 compared with the intake of the low-ALA control diet, indicating the existence of independent favourable physiological effects of ALA during weight loss.

Key words: $\boldsymbol{\alpha}$-Linolenic acid: Inflammation: Blood pressure: Endothelial function: Energy restriction

The metabolic syndrome, a concurrence of at least three of the parameters such as overweight and abdominal fat distribution, dyslipidaemia (elevated TAG and/or reduced HDL-cholesterol), arterial hypertension, impaired glucose metabolism, and chronic low-grade inflammation, has gained awareness and interest. It is directly correlated with the development and progression of atherosclerotic vascular disease as well as with type 2 diabetes mellitus, and identifies individuals at a higher risk of CVD than the general population ${ }^{(1-3)}$. Importantly, the close relationship between the metabolic syndrome, endothelial dysfunction and hypertension is linked to elevated cardiovascular risk, coronary artery disease and mortality ${ }^{(4-6)}$.
Recently, a number of circulating endothelial and inflammatory markers have been identified and implicated in the pathogenesis of atherosclerosis and $\mathrm{CVD}^{(7,8)}$. Cellular adhesion molecules including intercellular adhesion molecule-1, vascular cell adhesion molecule-1 and endothelial selectin are generally accepted to be associated with the pathogenesis of atherosclerosis and are the indicators of endothelial dysfunction preceding clinical events ${ }^{(8)}$. These endothelial markers are elevated in obesity and in patients with metabolic syndrome traits ${ }^{(8-10)}$. Similarly, inflammatory markers including high-sensitivity (hs) C-reactive protein (CRP), TNF- $\alpha$ and IL-6 are associated with atherosclerosis,

\footnotetext{
Abbreviations: ADMA, asymmetric dimethylarginine; ALA, $\alpha$-linolenic acid; BP, blood pressure; CRP, C-reactive protein; hs, high sensitivity; sE-selectin, soluble endothelial selectin; sICAM-1, soluble intercellular adhesion molecule-1; sVCAM-1, soluble vascular cell adhesion molecule-1; YKL-40, human cartilage glycoprotein 39 or chitinase-3-like protein 1.
}

*Corresponding author: S. Egert, fax +49228 733217, email s.egert@uni-bonn.de

† Both authors contributed equally to this work. 
and have also been shown to be elevated in obesity ${ }^{(11-13)}$. YKL-40, also known as human cartilage glycoprotein 39 or chitinase-3-like protein 1 , is an inflammatory glycoprotein involved in endothelial dysfunction by promoting chemotaxis, cell attachment and migration, reorganisation and tissue remodelling in response to endothelial damage ${ }^{(14)}$. Elevated circulating YKL-40 levels have been found to be associated with cardiovascular as well as all-cause mortality ${ }^{(14-16)}$. In addition, previous studies have reported that patients with type 2 diabetes mellitus exhibit elevated plasma levels of YKL-40 compared with healthy control subjects ${ }^{(17,18)}$. Recent studies have suggested that YKL-40 might act not only as a potential biomarker for endothelial dysfunction, but also for atherosclerosis, insulin resistance and type 2 diabetes mellitus ${ }^{(19)}$.

A large body of epidemiological data and evidence from randomised controlled human trials has demonstrated cardioprotective effects of the marine $n-3$ fatty acids EPA and $\mathrm{DHA}^{(20-22)}$. For example, EPA and DHA have been shown to reduce serum TAG concentrations, to lower arterial blood pressure (BP), to reduce inflammation, and to improve vascular endothelial function ${ }^{(23-27)}$. It is largely unknown whether the plant-derived $\alpha$-linolenic acid (ALA) has a significant preventive potential, too. Possible cardioprotective and anti-inflammatory benefits of dietary ALA have been reviewed recently ${ }^{(28-30)}$. Potential effects have been examined with different ALA dosages and under isoenergetic and thus weight-stable study conditions. To the best of our knowledge, there is no human trial investigating ALA-specific cardioprotective effects during energy restriction and body-weight loss. Therefore, in the present study, we examined, for the first time, the effects of an energy-restricted diet enriched with ALA on the biomarkers of vascular function and inflammation in patients with metabolic syndrome traits. We have previously reported the beneficial effects of this diet regimen on the parameters of lipid and glucose metabolism ${ }^{(31)}$. A range of subject baseline parameters, diet intake characteristics and also some results are re-presented here, in order to better relate them to the newly presented biomarkers.

\section{Patients and methods}

\section{Patients}

Details of the study design, dietary interventions, and patient recruitment, enrolment and randomisation have been described previously ${ }^{(31)}$. In brief, 150 interested volunteers aged 18-70 years with overweight attended a screening examination that included physical assessments (height, body weight, BP, waist and hip circumferences), clinical assessments (e.g. serum lipids and lipoproteins, glucose), medical history and a dietary questionnaire.

Participants who had the following traits of the metabolic syndrome were included: central obesity (waist circumference $\geq 94 \mathrm{~cm}$ for men and $\geq 80 \mathrm{~cm}$ for women) plus two of the following criteria: (1) fasting serum TAG concentrations of $\geq 1.7 \mathrm{mmol} / \mathrm{l}$; (2) reduced serum HDL-cholesterol $(<1.03 \mathrm{mmol} / 1 \mathrm{in} \mathrm{men;}<1.29 \mathrm{mmol} / 1$ in women); (3) elevated
BP (systolic $\geq 130 \mathrm{mmHg}$; diastolic $\geq 85 \mathrm{mmHg}$ ); (4) fasting plasma glucose $\geq 6.5 \mathrm{mmol} / \mathrm{l}^{(31,32)}$. Exclusion criteria were as follows: smoking; insulin-dependent diabetes mellitus; liver, gastrointestinal or inflammatory diseases; history of cardiovascular events; use of anti-obesity medications or anti-inflammatory drugs; cancer; pregnancy or breast-feeding; alcohol abuse; necessity for a medically supervised diet.

A total of ninety-five patients (thirty male and sixty-five female) were included in the study. Of these, thirteen patients dropped out due to different reasons, and one participant was retrospectively excluded because of multiple drug changes. Thus, the final analysis included eighty-one patients (twentysix male and fifty-five female) ${ }^{(31)}$. Baseline characteristics are presented in Table 1.

All study procedures were approved by the ethics committee of the Ruhr-University Bochum, located at HDZ NRW in Bad Oeynhausen, Germany, and were in compliance with the Declaration of Helsinki. All participants provided informed written consent. The study was registered at http://www. germanctr.de/ and http://apps.who.int/trialsearch/ as DRKS 00006232 .

\section{Study design and dietary intervention}

The study was a randomised controlled dietary intervention study and was conducted under outpatient conditions over a 26-week intervention period ${ }^{(31)}$. In a parallel design, patients were randomised to an energy-restricted diet (see below) enriched with ALA (ALA diet; approximately $3.4 \mathrm{~g}$ ALA/d) or an energy-restricted control diet (approximately $0.9 \mathrm{~g} \mathrm{ALA} / \mathrm{d}$ ) (Table 2). The principal sources of dietary fat during the inter-

Table 1. Baseline characteristics of the overweight-to-moderately obese men and women who completed the study ${ }^{(31) *}$

(Mean values and standard deviations)

\begin{tabular}{|c|c|c|c|c|}
\hline & \multicolumn{2}{|c|}{$\begin{array}{l}\alpha \text {-Linolenic } \\
\text { acid }(n 40)\end{array}$} & \multicolumn{2}{|c|}{$\begin{array}{c}\text { Control } \\
(n 41)\end{array}$} \\
\hline & Mean & SD & Mean & SD \\
\hline Age (years) & $52 \cdot 3$ & $10 \cdot 6$ & $50 \cdot 3$ & $9 \cdot 8$ \\
\hline Body weight (kg) & $97 \cdot 3$ & $19 \cdot 7$ & 99.4 & $16 \cdot 2$ \\
\hline BMI $\left(\mathrm{kg} / \mathrm{m}^{2}\right)$ & $33 \cdot 4$ & $4 \cdot 8$ & $35 \cdot 2$ & $5 \cdot 1$ \\
\hline \multicolumn{5}{|l|}{ Waist circumference $(\mathrm{cm})$} \\
\hline Men & $98 \cdot 1$ & $9 \cdot 6$ & $111 \cdot 1$ & $10 \cdot 0$ \\
\hline Women & $114 \cdot 1$ & $10 \cdot 5$ & $104 \cdot 8$ & $12 \cdot 1$ \\
\hline \multicolumn{5}{|l|}{ Fat mass (\%) } \\
\hline Men & 35.9 & $4 \cdot 1$ & $34 \cdot 3$ & $5 \cdot 2$ \\
\hline Women & $44 \cdot 8$ & $4 \cdot 6$ & $47 \cdot 1$ & $4 \cdot 3$ \\
\hline Systolic blood pressure (mmHg) & $142 \cdot 4$ & $18 \cdot 6$ & $140 \cdot 1$ & $12 \cdot 4$ \\
\hline Diastolic blood pressure $(\mathrm{mmHg})$ & $91 \cdot 8$ & $11 \cdot 8$ & $90 \cdot 2$ & $7 \cdot 7$ \\
\hline Fasting plasma glucose $(\mathrm{mmol} / \mathrm{l})$ & $5 \cdot 76$ & $1 \cdot 32$ & $5 \cdot 80$ & 1.00 \\
\hline Fasting plasma insulin (pmol/l) & $90 \cdot 7$ & $41 \cdot 1$ & 98.9 & $73 \cdot 7$ \\
\hline Fasting total cholesterol ( $\mathrm{mmol} / \mathrm{l})$ & 5.43 & 0.88 & 5.49 & 1.09 \\
\hline Fasting LDL-cholesterol (mmol/l) & 3.42 & 0.82 & 3.49 & 0.92 \\
\hline \multicolumn{5}{|l|}{ Fasting HDL-cholesterol (mmol/l) } \\
\hline Men & $1 \cdot 12$ & $0 \cdot 18$ & 1.32 & 0.41 \\
\hline Women & 1.50 & $0 \cdot 26$ & 1.47 & 0.30 \\
\hline Fasting TAG (mmol/l) & 1.94 & $1 \cdot 13$ & 1.64 & 1.02 \\
\hline ApoB $(g / l)$ & 0.92 & $0 \cdot 21$ & 0.93 & 0.23 \\
\hline ApoA1 (g/l) & 1.57 & $0 \cdot 28$ & 1.57 & 0.26 \\
\hline
\end{tabular}

* The two groups did not differ significantly with regard to any of these variables $(P>0.05$; independent-samples $t$ tests or Mann-Whitney $U$ test). 
Table 2. Composition of the habitual diet and the study diets*

(Mean values and standard deviations)

\begin{tabular}{|c|c|c|c|c|c|c|}
\hline & \multicolumn{2}{|c|}{$\begin{array}{l}\text { Habitual diet } \\
\qquad(n 81)\end{array}$} & \multicolumn{2}{|c|}{$\operatorname{ALA}(n 40)$} & \multicolumn{2}{|c|}{ Control (n 41) } \\
\hline & Mean & SD & Mean & SD & Mean & SD \\
\hline Energy intake (MJ/d) & $9 \cdot 1$ & $2 \cdot 8$ & $6 \cdot 8$ & 1.5 & $6 \cdot 7$ & $1 \cdot 2$ \\
\hline Protein (\% of energy) & $17 \cdot 5$ & $3 \cdot 3$ & $19 \cdot 5$ & $3 \cdot 8$ & $19 \cdot 0$ & 2.6 \\
\hline Carbohydrates (\% of energy) & 43.9 & $6 \cdot 9$ & $41 \cdot 3$ & $4 \cdot 3$ & $42 \cdot 4$ & $3 \cdot 6$ \\
\hline Fat $(\%$ of energy) $\dagger$ & $37 \cdot 3$ & $6 \cdot 2$ & 37.9 & $4 \cdot 5$ & $37 \cdot 8$ & 3.5 \\
\hline SFA (\% of energy) & $15 \cdot 4$ & 3.5 & $9 \cdot 8$ & $2 \cdot 2$ & $10 \cdot 7$ & 1.5 \\
\hline MUFA (\% of energy) & $13 \cdot 6$ & $2 \cdot 7$ & $18 \cdot 1$ & $2 \cdot 6$ & $19 \cdot 3$ & $2 \cdot 4$ \\
\hline PUFA (\% of energy) & $5 \cdot 2$ & 1.6 & $7 \cdot 8$ & 1.5 & 5.5 & 0.7 \\
\hline$A L A(g / d)$ & 1.61 & 0.77 & 3.35 & 0.65 & 0.85 & 0.16 \\
\hline $\mathrm{LA}(\mathrm{g} / \mathrm{d})$ & $10 \cdot 1$ & 3.6 & $10 \cdot 2$ & $2 \cdot 2$ & $8 \cdot 6$ & $1 \cdot 4$ \\
\hline Dietary fibre (g/MJ) & $2 \cdot 6$ & 0.9 & $4 \cdot 1$ & 0.9 & $4 \cdot 1$ & 0.9 \\
\hline Energy density $(\mathrm{kJ} / \mathrm{g}) \ddagger$ & $5 \cdot 9$ & $1 \cdot 2$ & 4.5 & 0.7 & $4 \cdot 6$ & 0.8 \\
\hline
\end{tabular}

vention period were either refined rapeseed oil (ALA, 7\% of total fatty acids) and a rapeseed oil-based commercial margarine for the ALA diet (Goldina; ALA, 6\% of total fatty acids), or refined olive oil (Henry Lamotte Oils; ALA, 0.5\% of total fatty acids) and a commercial olive oil-based margarine (ALA, 0.5\% of total fatty acids, mOlivo; Vitaquell) in the control group. The plant oils and the margarines were used for the preparation of all meals and snacks. The ALA diet was identical to the control diet in all respects except the principal sources of dietary fat. The consumption of fish, fish oil capsules and foods enriched with $n$ - 3 fatty acids was not allowed in both intervention groups to ensure that the diets were free of long-chain $n$-3 fatty acids ${ }^{(31)}$.

Before the intervention period, the patients were instructed to complete a $3 \mathrm{~d}$ dietary record for estimating their habitual energy and nutrient intake. During the intervention period (weeks 12 and 26), two further dietary records were completed for assessing the adherence to the prescribed diets. The dietary records as well as the study diets were calculated using the computer-based nutrient calculation program EBISpro (University of Hohenheim) based on the German Nutrient Data Base Bundeslebensmittelschlüssel, version II.3 (Max Rubner-Institut).

All patients received detailed instructions about the prescribed diets, instructions for food preparation and special recipes for use of the study oils and margarines. In addition, patients were provided with kitchen scales for weighing the prescribed food amounts and with a measuring cup for the daily oil amount. A nutritionist was in close and regular contact with the patients. Counselling about lifestyle, dietary behaviour and physical activity was identical for both intervention groups ${ }^{(31)}$.

The goal for body-weight reduction during the intervention period was $5-10 \%$ of the baseline body weight. To achieve this goal, hypoenergetic diets with a mean daily energy deficit in the range of $2 \cdot 0-3 \cdot 3 \mathrm{MJ}$ were calculated. On the basis of the expected energy expenditure of the patients, they were assigned to one of four energy intake levels $(5 \cdot 7,6 \cdot 3,6 \cdot 9$ or $7 \cdot 8 \mathrm{MJ} / \mathrm{d})^{(31)}$. The intervention diets were calculated with $42 \%$ of total energy as carbohydrates, $20 \%$ of energy as protein and $38 \%$ as dietary fat (Table 2). The main components of both diets were low-fat foodstuffs, e.g. whole-grain bread and cereals, vegetables, fruits, lean meat, skimmed milk and low-fat dairy products as described previously ${ }^{(31)}$.

\section{Measurements of anthropometry, body composition and arterial blood pressure}

Body height was determined using a wall-mounted stadiometer to the nearest $0.5 \mathrm{~cm}$ during the first examination. Waist circumference was measured to the nearest $0.5 \mathrm{~cm}$ midway between the lowest rib and the iliac crest, while the participant was at minimal respiration. Body composition was determined by bioelectric impedance analysis (Maltron International Limited). BP measurements were obtained with a fully automated BP monitor (Bosch+Sohn) as described previously ${ }^{(31)}$.

\section{Blood sampling}

Venous blood samples were obtained at baseline (week 0) and after 12 and 26 weeks of consuming the energy-restricted diets. All samples were taken after an overnight fast of at least $10 \mathrm{~h}$ under standardised conditions. Serum and plasma were obtained by centrifugation at $1500 \mathrm{~g}$ for $20 \mathrm{~min}$ at $4^{\circ} \mathrm{C}$, and stored at $-80^{\circ} \mathrm{C}$ until analysis. All laboratory measurements were performed blinded, without any knowledge of the intervention group. Serum samples were used for the determination of lipid parameters, insulin, glucose, hs-CRP, hs-TNF- $\alpha$, hs-IL- 6 , soluble vascular cell adhesion molecule-1 (sVCAM-1), soluble intercellular adhesion molecule-1 (sICAM-1), soluble endothelial selectin (sE-selectin), asymmetric dimethylarginine (ADMA) and YKL-40. EDTA plasma was used for the measurements of big endothelin-1; citrate plasma was used for the analysis of fibrinogen. 
Measurements of serum lipid, glucose, insulin and high-sensitivity C-reactive protein concentrations

Fasting serum concentrations of total cholesterol, LDL-cholesterol, HDL-cholesterol, TAG, apoB and apoA1, glucose and insulin were measured using the autoanalyser Architect System (ci8200 series; Abbott Diagnostics) as described previously $^{(31)}$. Serum concentrations of hs-CRP were determined using an automated high-sensitivity immunoturbidimetric test.

Measurements of fibrinogen, high-sensitivity TNF- $\alpha$, highsensitivity IL-6, soluble vascular cell adhesion molecule-1, soluble intercellular adhesion molecule-1, soluble endothelial selectin, asymmetric dimethylarginine, big endothelin-1 and YKL-40 concentrations

Plasma fibrinogen was measured by the Clauss assay using the Sysmex CA-7000 autoanalyser (Siemens Healthcare Diagnostics). Commercial ELISA assays were used to measure serum concentrations of hs-IL-6 (R\&D Systems Europe), hs-TNF- $\alpha$ (R\&D Systems), sE-selectin (R\&D Systems), sVCAM-1 (R\&D Systems), sICAM-1 (R\&D Systems), ADMA (Immundiagnostik AG), YKL-40 (Quidel) and plasma concentrations of big endothelin-1 (Biomedica). The samples were analysed in duplicate with a commercial microplate reader (Tecan). All inter-assay CV were $\leq 10 \%$.

\section{Statistical analyses}

Statistical analyses were performed using the Statistical Package for the Social Sciences, version 11.5 (SPSS, Inc.). The distribution of variables was analysed by checking histograms and normal plots of the data, and normality was tested by means of Kolmogorov-Smirnov and Shapiro-Wilk tests. Baseline characteristics of the groups were compared by means of independent-samples $t$ tests or Mann-Whitney $U$ test. Changes in anthropometric parameters and blood parameters were compared by repeated-measures ANOVA, with the data of weeks 0,12 and 26 as the three levels of the within-subject factor (time) and treatment (ALA $v$. control) as the between-subject factor. Repeated-measures ANOVA were conducted with log-transformed variables if the residuals were non-normally distributed, which was the case for hs-CRP, sVCAM-1, sICAM-1, sE-Selectin, YKL- 40 , hs-TNF- $\alpha$ and hs-IL-6. All tests were two-tailed, and $P$ values $\leq 0.05$ were considered significant. Pearson's correlation coefficient was used to evaluate the relationships between different variables.

\section{Results}

\section{Body weight, fat mass and arterial blood pressure}

As reported previously, body weight decreased throughout the study by 7.8 (SE 6.2) $\mathrm{kg}$ in the ALA group and by 6.0 (SE 4.8) $\mathrm{kg}$ in the control group, changes that were significant overall but that did not differ significantly between the treatment groups $(P<0.001$ for time; $P=0.155$ for time $\times$ treatment interaction; Fig. 1) ${ }^{(31)}$. Body fat mass decreased by a mean of $5.8(\operatorname{se~} 4.5) \mathrm{kg}$ in the ALA group and by $4.2(\mathrm{SE} 4.4) \mathrm{kg}$ in the control group.
This change was significant overall ( $P<0.001$ for time), but did not differ significantly between the two intervention groups ( $P=0.263$ for time $\times$ treatment interaction; data not shown). In addition, systolic and diastolic BP significantly decreased throughout the study (systolic BP, $-10 \cdot 0$ ( $\mathrm{SE} \mathrm{13.0)} \mathrm{mmHg}$ in the ALA group $v$. -8.0 (SE 14.6) $\mathrm{mmHg}$ in the control group, $P<0.001$ for time; diastolic BP, -8.4 (SE 9.3) $\mathrm{mmHg}$ in the ALA group $v .-4.4$ (SE 6.6) $\mathrm{mmHg}$ in the control group, $P<0.001$ for time). The latter was more pronounced for the ALA group when compared with the control group $(P=0.026$ for time $\times$ treatment interaction). Resting systolic and diastolic BP at baseline and the changes in systolic and diastolic BP from baseline to after intervention were correlated with the individuals with higher baseline BP, demonstrating greater reductions in systolic and diastolic BP in response to the intervention diets (total study group: systolic BP, $r-0.567, P<0.001$; diastolic $\mathrm{BP}, r-0.489$, $P<0 \cdot 001)$. In addition, changes in body fat mass correlated with changes in systolic $(r 0 \cdot 26, P<0.05)$ and diastolic BP $(r$ $0.362, P<0 \cdot 001)$

\section{Biomarkers of inflammation and endothelial function}

Serum hs-CRP, hs-TNF- $\alpha$ and hs-IL-6 concentrations decreased significantly over time, but there was no effect of treatment group on this change (Table 3). The plasma concentrations of fibrinogen neither changed significantly throughout the study nor differed in any way between the two dietary groups (Table 3). The serum concentrations of sVCAM-1 and plasma big endothelin-1 slightly increased throughout the study, with no effect of treatment group on these changes (Table 3). In contrast, serum sICAM-1, sE-selectin and ADMA decreased significantly over time, but decreases were independent of the intervention (Table 3). During both treatments, serum YKL-40 concentration significantly decreased over time. The decrease in the concentration of YKL- 40 was significantly more pronounced in the ALA group than in the control group (Table 3).

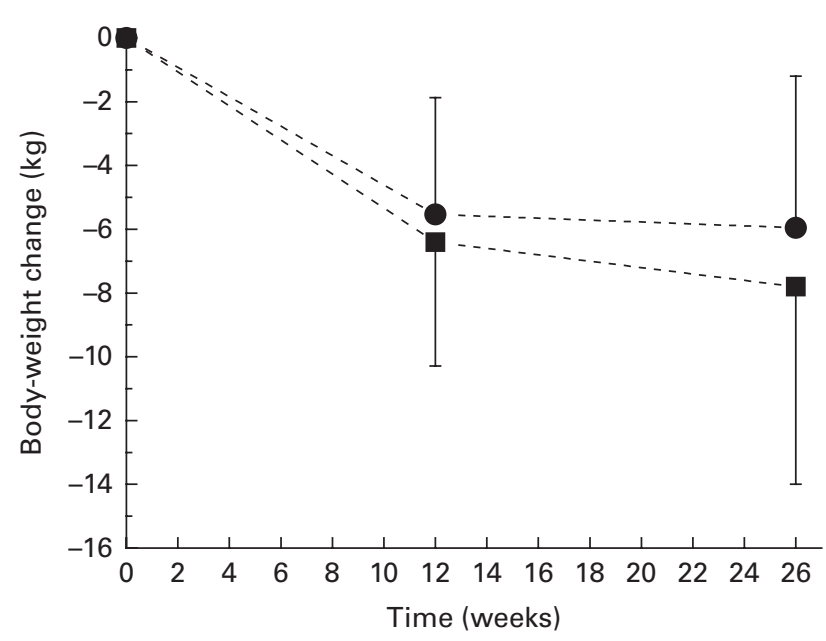

Fig. 1. Body-weight changes during 26 weeks of energy restriction with two different diets: $\alpha$-linolenic acid (ALA) diet (----, $n$ 40); control diet low in ALA $(-\bullet--, n 41)$. Values are means, with their standard errors represented by vertical bars. There was a significant effect for time $(P<0.001)$, but no significant diet $\times$ time interaction effect $(P=0.155)$. 
Table 3. Fasting biomarkers of inflammation and endothelial function in overweight-to-moderately obese men and women at baseline (week 0 ) and after 12 and 26 weeks of consuming an energy-restricted diet rich in $\alpha$-linolenic acid (ALA; $n$ 38) v. a control diet low in ALA ( $n$ 39)

(Mean values and standard deviations)

\begin{tabular}{|c|c|c|c|c|c|c|c|c|c|c|}
\hline & \multicolumn{2}{|c|}{ Week 0} & \multicolumn{2}{|c|}{ Week 12} & \multicolumn{2}{|c|}{ Week 26} & \multicolumn{2}{|c|}{ Change } & \multicolumn{2}{|c|}{$P($ RM-ANOVA) } \\
\hline & Mean & SD & Mean & SD & Mean & SD & Mean & SD & Time & Timextreatment \\
\hline \multicolumn{11}{|c|}{ Serum hs-CRP (mg/l) } \\
\hline ALA & 3.78 & 4.66 & 3.07 & 3.50 & $2 \cdot 70$ & 3.28 & -1.08 & 2.99 & $<0.001$ & 0.279 \\
\hline Control & 5.15 & 5.81 & 3.63 & 2.97 & 4.03 & 3.59 & $-1 \cdot 12$ & 5.40 & & \\
\hline \multicolumn{11}{|c|}{ Serum hs-TNF- $\alpha$ (pg/ml) } \\
\hline ALA & 1.49 & 0.91 & 1.42 & 0.83 & 1.32 & 0.66 & -0.17 & 0.47 & $<0.001$ & 0.794 \\
\hline Control & 1.36 & 0.45 & 1.27 & 0.44 & 1.21 & 0.44 & -0.15 & 0.32 & & \\
\hline \multicolumn{11}{|c|}{ Serum hs-IL-6 (pg/ml) } \\
\hline ALA & 1.33 & 0.69 & 1.12 & 0.45 & 1.16 & 0.89 & -0.17 & 0.92 & $<0.01$ & 0.838 \\
\hline Control & 1.34 & 0.81 & 1.34 & 0.85 & 1.11 & 0.51 & -0.23 & 0.77 & & \\
\hline \multicolumn{11}{|c|}{ Plasma fibrinogen $(\mu \mathrm{mol} / \mathrm{l})$} \\
\hline ALA & 8.40 & 2.07 & 8.64 & 1.77 & 8.65 & 1.59 & 0.25 & 1.78 & 0.094 & 0.242 \\
\hline Control & 9.14 & 2.85 & 9.07 & 2.25 & 9.95 & $2 \cdot 23$ & 0.81 & 2.86 & & \\
\hline \multicolumn{11}{|c|}{ Serum sVCAM-1 (ng/ml) } \\
\hline ALA & $755 \cdot 6$ & $155 \cdot 1$ & 773.6 & $158 \cdot 8$ & 794.5 & $195 \cdot 1$ & 38.9 & $120 \cdot 0$ & $<0.01$ & 0.526 \\
\hline Control & $730 \cdot 1$ & $252 \cdot 1$ & 774.4 & 270.5 & 773.4 & 273.8 & 43.3 & 118.5 & & \\
\hline \multicolumn{11}{|c|}{ Serum sICAM-1 (ng/ml) } \\
\hline ALA & $269 \cdot 4$ & $61 \cdot 1$ & 247.6 & 54.1 & 249.6 & $57 \cdot 3$ & -19.9 & $46 \cdot 2$ & $<0.001$ & 0.328 \\
\hline Control & $227 \cdot 6$ & $41 \cdot 7$ & $218 \cdot 8$ & 44.9 & $218 \cdot 2$ & 40.6 & -9.4 & $28 \cdot 0$ & & \\
\hline \multicolumn{11}{|c|}{ Plasma big endothelin-1 (fmol/ml) } \\
\hline ALA & 0.647 & 0.213 & 0.658 & 0.153 & 0.686 & 0.182 & 0.039 & 0.181 & $<0.05$ & 0.406 \\
\hline Control & 0.566 & 0.206 & 0.557 & 0.172 & 0.638 & 0.303 & 0.073 & 0.224 & & \\
\hline \multicolumn{11}{|c|}{ Serum sE-selectin (ng/ml) } \\
\hline ALA & $49 \cdot 7$ & 26.5 & $37 \cdot 7$ & $17 \cdot 6$ & 39.4 & $18 \cdot 4$ & $-10 \cdot 4$ & $13 \cdot 6$ & $<0.001$ & 0.063 \\
\hline Control & $48 \cdot 0$ & $19 \cdot 4$ & 40.5 & $17 \cdot 1$ & $42 \cdot 8$ & $17 \cdot 7$ & $-5 \cdot 2$ & 6.8 & & \\
\hline \multicolumn{11}{|c|}{ Serum ADMA ( $\mu \mathrm{mol} / \mathrm{l})$} \\
\hline ALA & 0.458 & 0.068 & 0.438 & 0.065 & 0.433 & 0.061 & -0.026 & 0.055 & $<0.01$ & 0.726 \\
\hline Control & 0.422 & 0.065 & 0.412 & 0.059 & 0.405 & 0.068 & -0.017 & 0.072 & & \\
\hline \multicolumn{11}{|c|}{ Serum YKL-40 (ng/ml) } \\
\hline ALA & 78.9 & $41 \cdot 6$ & $61 \cdot 0$ & 33.1 & $59 \cdot 3$ & 24.9 & $-19 \cdot 6$ & 31.3 & $<0.001$ & $<0.05$ \\
\hline Control & 74.3 & $76 \cdot 4$ & 68.9 & 44.2 & $65 \cdot 2$ & 44.0 & -9.1 & $46 \cdot 2$ & & \\
\hline
\end{tabular}

RM-ANOVA, repeated-measures ANOVA; hs, high sensitivity; CRP, C-reactive protein; sVCAM-1, soluble vascular cell adhesion molecule-1; sICAM-1, soluble intercellular adhesion molecule-1; sE-selectin, soluble endothelial selectin; ADMA, asymmetric dimethylarginine; YKL-40, human cartilage glycoprotein 39 or chitinase-3-like protein 1. 
Serum concentrations of hs-CRP, hs-TNF- $\alpha$, hs-IL-6, sICAM-1, sE-selectin, ADMA and YKL-40 at baseline and the changes in these biomarkers from baseline to after intervention were significantly correlated with the individuals with higher baseline concentrations, demonstrating greater reductions in these biomarkers in response to the intervention diets (hs-CRP, $r$-0.756; hs-TNF- $\alpha, r-0.632$; hs-IL-6, $r-0.601$; sICAM-1, $r-0.448$; sE-selectin, $r-0.649$; ADMA, $r-0.518$; YKL-40, $r-0.834 ; P<0.001$ for all such values). In addition, changes in body fat mass correlated with changes in the concentrations of sICAM-1 $(r$ 0.284, $P<0.05)$, sE-selectin $(r 0.411, P<0.001)$, hs-CRP $(0 \cdot 377, P<0 \cdot 001)$ and YKL-40 $(r 0.324, P<0 \cdot 01)$.

Baseline systolic and diastolic BP did not significantly correlate with the biomarkers of inflammation and endothelial function. In addition, changes in BP variables did not significantly correlate with changes in the biomarkers of inflammation and endothelial function.

\section{Discussion}

The aim of the present randomised controlled dietary intervention study in overweight and moderately obese patients with metabolic syndrome traits was to investigate the effects of an energy-restricted diet rich in ALA on vascular function and systemic inflammation. After a 26-week intervention period, a distinct improvement in the biomarkers of endothelial function, resting $\mathrm{BP}$ and inflammation in the ALA group as well in the control group was detected. The high ALA intake led to a more pronounced reduction in serum YKL-40 concentration and diastolic BP compared with the intake of low-ALA control diet, indicating that there may be independent favourable physiological effects of ALA during weight loss. To our knowledge, this type of investigation has not been reported previously.

The observed decrease in systolic and diastolic BP during loss in body fat mass is similar to the results from previous weight-loss studies using different energy-restricted diets ${ }^{(33-35)}$. A metaanalysis by Neter et $a l{ }^{(36)}$ of twenty-five randomised controlled trials showed a reduction in systolic and diastolic BP of -1.05 and $-0.92 \mathrm{mmHg} / \mathrm{kg}$ body-weight loss, respectively. Although the exact mechanism of the relationship between obesity and elevated BP and the effect of body-weight loss on BP is unknown, there are several plausible biological pathways. For example, the adipose renin-angiotensin-aldosterone system is overactivated in obese individuals, and renin activity and aldosterone concentrations are higher in obese than in lean subjects ${ }^{(37)}$. In addition, activity of the sympathetic nervous system is increased in hypertensive, obese individuals, which could induce obesityrelated renal effects ${ }^{(38)}$. Decreased insulin sensitivity and hyperinsulinaemia as part of the metabolic syndrome might also form an essential link between obesity, sympathetic nervous activation and hypertension, and energy restriction has been shown to improve insulin sensitivity ${ }^{(37,39)}$. As published previously ${ }^{(31)}$, fasting serum concentrations of insulin and intact proinsulin were significantly reduced in both dietary groups in the present study. The improvement of the metabolic situation in terms of insulin resistance and homoeostatic model assessment of insulin resistance (HOMA-IR) may have an impact on YKL-40 levels, too.

The magnitude of the hypotensive effect observed in the present study and previously during body-weight loss ${ }^{(36)}$ is certainly clinically relevant, and is expected to considerably reduce the risk of CVD in our patients. For example, a reduction in systolic $\mathrm{BP}$ of $10 \mathrm{mmHg}$ - comparable to the present results - lowered the risk of myocardial infarction by $21 \%$ in the UK Prospective Diabetes Study ${ }^{(40)}$.

A significantly more pronounced decline in diastolic BP was observed after the intake of the ALA-rich diet than after the intake of the control diet. We speculate from these results that there might be an independent effect of ALA on BP. This finding was confirmed in a Greek study in normotensive, dyslipidaemic patients and under isoenergetic study conditions with an ALA intake of $8 \mathrm{~g} / \mathrm{d}$, which led to significant lower resting systolic and diastolic BP compared with a high intake of linoleic $\operatorname{acid}^{(41)}$. In contrast, other human trials in subjects with the risk of CVD did not report a BPlowering effect of $\mathrm{ALA}^{(42-44)}$. The physiological mechanisms by which dietary ALA might lower BP are not well understood. It is assumed that the long-chain $n$ - 3 dietary fatty acids EPA and DHA at high doses $(\geq 3 \mathrm{~g} / \mathrm{d})$ can modulate BP mainly through their effects on the renin-angiotensin-aldosterone system, their effects on peripheral sympathetic tone, the reduction in plasma viscosity, the production of endothelial $\mathrm{NO}$ and the production of 3-series PG with vasoactive effects $^{(45)}$. ALA can be converted to EPA and DHA by elongation and desaturation, but the extent of this conversion is not clear and at best very limited ${ }^{(46,47)}$. It, therefore, remains unclear whether the BP-lowering effects during the intake of an ALA diet can be ascribed to dietary ALA, or through its conversion to the long-chain $n$-3 fatty acids EPA and/or DHA.

In the present study, a number of markers of endothelial function and inflammation (e.g. sICAM-1, sE-selectin, hs-CRP, hs-TNF- $\alpha$, hs-IL-6) were improved probably mainly as a result of body fat loss during the two energy-restricted diets accounting for decreased endothelial cell activation. Similar findings have been reported in several previous studies after weight loss ${ }^{(33-35,48)}$. For example, Ziccardi et al. ${ }^{(48)}$ found reductions in the concentrations of IL-6, TNF- $\alpha$, sVCAM-1 and sICAM-1 following a body-weight loss of $10 \mathrm{~kg}(10 \%)$ in obese women. The levels of SICAM-1 predict future cardiovascular events in healthy subjects, and the reduction in the concentrations of these markers leads to a reduction in the risk of $\mathrm{CVD}^{(49)}$. We found small but significant increases in the concentrations of sVCAM-1 and big endothelin-1 (Table 3), which we are unable to explain. However, we believe that these small increases are unlikely to be biologically important.

Diet-induced body-weight loss was able to significantly reduce the circulating YKL-40 concentrations in our overweight-to-obese patients with metabolic syndrome traits. Similar results have been reported recently in obese patients with type 2 diabetes mellitus ${ }^{(50)}$. In addition, we found a positive association between YKL-40 and body fat. The biological function of YKL-40 is not yet clear; however, the present results strengthen the view for an involvement of YKL-40 in low-grade inflammation associated with 
abdominal fat distribution and the development of the metabolic syndrome. We observed a significantly more pronounced decrease in circulating YKL-40 concentration after the intake of the ALA-rich diet than after the intake of the control diet. To the best of our knowledge, this finding has not been reported previously. We speculate from the present results that there might be an independent effect of dietary ALA on the regulation of YKL- 40 .

In the present study, endothelial-derived adhesion molecules did not correlate with BP, emphasising that factors regulating endothelial function differ at least in part from those regulating BP.

In conclusion, the present study indicates that in overweightto-obese patients with metabolic syndrome traits, both vascular function and inflammation are improved during body-weight loss. In addition, the ALA diet alone was associated with significant reductions in diastolic BP and serum YKL- 40 concentration. These effects of ALA may provide novel mechanisms by which ALA may affect vascular compliance.

\section{Acknowledgements}

The present study was supported by the Union for the Promotion of Oil and Protein Plants (UFOP) and the International Foundation for the Promotion of Nutrition Research and Nutrition Education (ISFE). UFOP and ISFE had no role in the design and analysis of the study or in the writing of this article.

The authors' contributions are as follows: U. W., B. S., S. E. and D. T. designed the research; Y. H. L.-B. and A. B. conducted the research; A. B. and S. E. analysed the data; S. E. wrote the manuscript; B. S. and U. W. had primary responsibility for the final content. All authors read and approved the final manuscript.

None of the authors has any conflict of interest to declare.

\section{References}

1. Alberti KG, Eckel RH, Grundy SM, et al. (2009) Harmonizing the metabolic syndrome: a joint interim statement of the International Diabetes Federation Task Force on Epidemiology and Prevention; National Heart, Lung, and Blood Institute; American Heart Association; World Heart Federation; International Atherosclerosis Society; and International Association for the Study of Obesity. Circulation 120, 1640-1645.

2. Wilson PW, D'Agostino RB, Parise H, et al. (2005) Metabolic syndrome as a precursor of cardiovascular disease and type 2 diabetes mellitus. Circulation 112, 3066-3072.

3. Grundy SM (2012) Pre-diabetes, metabolic syndrome, and cardiovascular risk. J Am Coll Cardiol 59, 635-643.

4. Di Pino A, Alagona C, Piro S, et al. (2012) Separate impact of metabolic syndrome and altered glucose tolerance on early markers of vascular injuries. Atherosclerosis 223, 458-462.

5. Niccoli G, Giubilato S, Di Vito L, et al. (2013) Severity of coronary atherosclerosis in patients with a first acute coronary event: a diabetes paradox. Eur Heart J 34, 729-741.

6. Suzuki T, Hirata K, Elkind MS, et al. (2008) Metabolic syndrome, endothelial dysfunction, and risk of cardiovascular events: the Northern Manhattan Study (NOMAS). Am Heart J 156, 405-410.
7. Hope SA \& Meredith IT (2003) Cellular adhesion molecules and cardiovascular disease. Part I. Their expression and role in atherogenesis. Intern Med J 33, 380-386.

8. Hope SA \& Meredith IT (2003) Cellular adhesion molecules and cardiovascular disease. Part II. Their association with conventional and emerging risk factors, acute coronary events and cardiovascular risk prediction. Intern Med J 33, 450-462.

9. Jacobs M, van Greevenbroek MM, van der Kallen CJ, et al. (2011) The association between the metabolic syndrome and peripheral, but not coronary, artery disease is partly mediated by endothelial dysfunction: the CODAM study. Eur J Clin Invest 41, 167-175.

10. Palomo IG, Jaramillo JC, Alarcon ML, et al. (2009) Increased concentrations of soluble vascular cell adhesion molecule-1 and soluble CD40L in subjects with metabolic syndrome. Mol Med Rep 2, 481-485.

11. Alexopoulos N, Katritsis D \& Raggi P (2014) Visceral adipose tissue as a source of inflammation and promoter of atherosclerosis. Atherosclerosis 233, 104-112.

12. Choi J, Joseph L \& Pilote L (2013) Obesity and C-reactive protein in various populations: a systematic review and meta-analysis. Obes Rev 14, 232-244.

13. Khosravi R, Ka K, Huang T, et al. (2013) Tumor necrosis factor- $\alpha$ and interleukin- 6 : potential interorgan inflammatory mediators contributing to destructive periodontal disease in obesity or metabolic syndrome. Mediators Inflamm, article ID $728987,1-6$.

14. Rathcke CN \& Vestergaard H (2009) YKL-40 - an emerging biomarker in cardiovascular disease and diabetes. Cardiovasc Diabetol $\mathbf{8}, 61$.

15. Kastrup J, Johansen JS, Winkel P, et al. (2009) High serum YKL-40 concentration is associated with cardiovascular and all-cause mortality in patients with stable coronary artery disease. Eur Heart J 30, 1066-1072.

16. Harutyunyan M, Gotze JP, Winkel P, et al. (2013) Serum YKL-40 predicts long-term mortality in patients with stable coronary disease: a prognostic study within the CLARICOR trial. Immunobiology 218, 945-951.

17. Nielsen AR, Erikstrup C, Johansen JS, et al. (2008) Plasma YKL-40: a BMI-independent marker of type 2 diabetes. Diabetes 57, 3078-3082.

18. Hempen M, Kopp HP, Elhenicky M, et al. (2009) YKL-40 is elevated in morbidly obese patients and declines after weight loss. Obes Surg 19, 1557-1563.

19. Rathcke CN, Johansen JS \& Vestergaard H (2006) YKL-40, a biomarker of inflammation, is elevated in patients with type 2 diabetes and is related to insulin resistance. Inflamm Res 55, 53-59.

20. Saravanan P, Davidson NC, Schmidt EB, et al. (2010) Cardiovascular effects of marine omega-3 fatty acids. Lancet $\mathbf{3 7 6}$, 540-550.

21. He K (2009) Fish, long-chain omega-3 polyunsaturated fatty acids and prevention of cardiovascular disease - eat fish or take fish oil supplement? Prog Cardiovasc Dis 52, 95-114.

22. Wang C, Harris WS, Chung M, et al. (2006) n-3 Fatty acids from fish or fish-oil supplements, but not $\alpha$-linolenic acid, benefit cardiovascular disease outcomes in primary- and secondary-prevention studies: a systematic review. Am J Clin Nutr 84, 5-17.

23. Egert $S$ \& Stehle P (2011) Impact of $n$-3 fatty acids on endothelial function: results from human interventions studies. Curr Opin Clin Nutr Metab Care 14, 121-131.

24. Jacobs B, Angelis-Schierbaum G, Egert S, et al. (2004) Individual serum triglyceride responses to high-fat and low-fat diets differ in men with modest and severe hypertriglyceridemia. J Nutr 134, 1400-1405. 
25. de Roos B, Mavrommatis Y \& Brouwer IA (2009) Long-chain $n-3$ polyunsaturated fatty acids: new insights into mechanisms relating to inflammation and coronary heart disease. Br J Pharmacol 158, 413-428.

26. Stirban A, Nandrean S, Gotting C, et al. (2010) Effects of $n-3$ fatty acids on macro- and microvascular function in subjects with type 2 diabetes mellitus. Am J Clin Nutr 91, 808-813.

27. Egert S, Kannenberg F, Somoza V, et al. (2009) Dietary $\alpha$-linolenic acid, EPA, and DHA have differential effects on LDL fatty acid composition but similar effects on serum lipid profiles in normolipidemic humans. J Nutr 139, 861-868.

28. Stark AH, Crawford MA \& Reifen R (2008) Update on $\alpha$-linolenic acid. Nutr Rev 66, 326-332.

29. Poudyal H, Panchal SK, Diwan V, et al. (2011) Omega-3 fatty acids and metabolic syndrome: effects and emerging mechanisms of action. Prog Lipid Res 50, 372-387.

30. Rodriguez-Leyva D, Dupasquier CM, McCullough R, et al. (2010) The cardiovascular effects of flaxseed and its omega-3 fatty acid, $\alpha$-linolenic acid. Can J Cardiol 26, 489-496.

31. Baxheinrich A, Stratmann B, Lee-Barkey YH, et al. (2012) Effects of a rapeseed oil-enriched hypoenergetic diet with a high content of $\alpha$-linolenic acid on body weight and cardiovascular risk profile in patients with the metabolic syndrome. Br J Nutr 108, 682-691.

32. Alberti KG, Zimmet P \& Shaw J (2005) The metabolic syndrome - a new worldwide definition. Lancet 366, 1059-1062

33. Wycherley TP, Brinkworth GD, Keogh JB, et al. (2010) Longterm effects of weight loss with a very low carbohydrate and low fat diet on vascular function in overweight and obese patients. J Intern Med 267, 452-461.

34. Clifton PM, Keogh JB, Foster PR, et al. (2005) Effect of weight loss on inflammatory and endothelial markers and FMD using two low-fat diets. Int $J$ Obes (Lond) 29, 1445-1451.

35. Keogh JB, Brinkworth GD, Noakes M, et al. (2008) Effects of weight loss from a very-low-carbohydrate diet on endothelial function and markers of cardiovascular disease risk in subjects with abdominal obesity. Am J Clin Nutr 87, $567-576$.

36. Neter JE, Stam BE, Kok FJ, et al. (2003) Influence of weight reduction on blood pressure: a meta-analysis of randomized controlled trials. Hypertension 42, 878-884.

37. Frigolet ME, Torres N \& Tovar AR (2013) The renin-angiotensin system in adipose tissue and its metabolic consequences during obesity. J Nutr Biochem 24, 2003-2015.

38. Masuo K, Rakugi H, Ogihara T, et al. (2010) Cardiovascular and renal complications of type 2 diabetes in obesity: role of sympathetic nerve activity and insulin resistance. Curr Diabetes Rev 6, 58-67.
39. Soare A, Weiss EP \& Pozzilli P (2014) Benefits of caloric restriction for cardiometabolic health, including type 2 diabetes mellitus risk. Diabetes Metab Res Rev 30, Suppl. 1, 41- 47 .

40. UK Prospective Diabetes Study Group (1998) Tight blood pressure control and risk of macrovascular and microvascular complications in type 2 diabetes: UKPDS 38. BMJ 317, 703-713.

41. Paschos GK, Magkos F, Panagiotakos DB, et al. (2007) Dietary supplementation with flaxseed oil lowers blood pressure in dyslipidaemic patients. Eur J Clin Nutr 61, 1201-1206.

42. Bemelmans WJ, Broer J, Feskens EJ, et al. (2002) Effect of an increased intake of $\alpha$-linolenic acid and group nutritional education on cardiovascular risk factors: the Mediterranean Alpha-linolenic Enriched Groningen Dietary Intervention (MARGARIN) study. Am J Clin Nutr 75, 221-227.

43. Finnegan YE, Minihane AM, Leigh-Firbank EC, et al. (2003) Plant- and marine-derived $n-3$ polyunsaturated fatty acids have differential effects on fasting and postprandial blood lipid concentrations and on the susceptibility of LDL to oxidative modification in moderately hyperlipidemic subjects. Am J Clin Nutr 77, 783-795.

44. Singer P, Berger I, Wirth M, et al. (1986) Slow desaturation and elongation of linoleic and $\alpha$-linolenic acids as a rationale of eicosapentaenoic acid-rich diet to lower blood pressure and serum lipids in normal, hypertensive and hyperlipemic subjects. Prostaglandins Leukot Med 24, 173-193.

45. Cabo J, Alonso R \& Mata P (2012) Omega-3 fatty acids and blood pressure. Br J Nutr 107, Suppl. 2, S195-S200.

46. Goyens PL, Spilker ME, Zock PL, et al. (2005) Compartmental modeling to quantify $\alpha$-linolenic acid conversion after longer term intake of multiple tracer boluses. J Lipid Res 46, 1474-1483.

47. Goyens PL, Spilker ME, Zock PL, et al. (2006) Conversion of $\alpha$-linolenic acid in humans is influenced by the absolute amounts of $\alpha$-linolenic acid and linoleic acid in the diet and not by their ratio. Am J Clin Nutr 84, 44-53.

48. Ziccardi P, Nappo F, Giugliano G, et al. (2002) Reduction of inflammatory cytokine concentrations and improvement of endothelial functions in obese women after weight loss over one year. Circulation 105, 804-809.

49. Ridker PM, Hennekens CH, Roitman-Johnson B, et al. (1998) Plasma concentration of soluble intercellular adhesion molecule 1 and risks of future myocardial infarction in apparently healthy men. Lancet 351, 88-92.

50. Catalan V, Gomez-Ambrosi J, Rodriguez A, et al. (2011) Increased circulating and visceral adipose tissue expression levels of YKL-40 in obesity-associated type 2 diabetes are related to inflammation: impact of conventional weight loss and gastric bypass. J Clin Endocrinol Metab 96, 200-209. 